\title{
Linear Optical Quantum Computing in a Single Spatial Mode
}

\author{
Peter C. Humphreys, ${ }^{1}$ Benjamin J. Metcalf, ${ }^{1}$ Justin B. Spring, ${ }^{1}$ Merritt Moore, ${ }^{1}$ Xian-Min Jin,,${ }^{1,2}$ Marco Barbieri, ${ }^{1}$ \\ W. Steven Kolthammer, ${ }^{1}$ and Ian A. Walmsley ${ }^{1}$ \\ ${ }^{1}$ Department of Physics, Clarendon Laboratory, University of Oxford, Oxford OX1 3PU, United Kingdom \\ ${ }^{2}$ Department of Physics, Shanghai Jiao Tong University, Shanghai 200240, People's Republic of China
} (Received 15 May 2013; published 9 October 2013)

\begin{abstract}
We present a scheme for linear optical quantum computing using time-bin-encoded qubits in a single spatial mode. We show methods for single-qubit operations and heralded controlled-phase (CPHASE) gates, providing a sufficient set of operations for universal quantum computing with the Knill-Laflamme-Milburn [Nature (London) 409, 46 (2001)] scheme. Our protocol is suited to currently available photonic devices and ideally allows arbitrary numbers of qubits to be encoded in the same spatial mode, demonstrating the potential for time-frequency modes to dramatically increase the quantum information capacity of fixed spatial resources. As a test of our scheme, we demonstrate the first entirely single spatial mode implementation of a two-qubit quantum gate and show its operation with an average fidelity of $0.84 \pm 0.07$.
\end{abstract}

PACS numbers: 03.67.Lx, 42.50.Dv, 42.50.Ex

Introduction.-Linear optics provides a promising platform for universal quantum computing [1-3]. Although logical gates can only be implemented probabilistically, Knill, Laflamme, and Milburn (KLM) have shown that they can be rendered deterministic by making use of ancillary resources, measurements, and feed-forward [1]. However, the overhead is large, and this presents one of the most significant challenges to the scalability of all proposed linear-optical quantum computing (LOQC) implementations $[2,3]$. To date, demonstrations of experimental schemes have mainly adopted spatial degrees of freedom for the manipulation of quantum states [2-9]. Consequently, scalable implementations of even few-qubit protocols in LOQC demand many spatial modes and complex routing networks with active switches, necessary to implement feed-forward [10].

Modern telecommunication suggests a promising alternative or complement to spatial schemes in its extensive use of time-frequency encodings. The same approach for quantum information and communication protocols naturally provides access to high dimensional Hilbert spaces [11-13] while maintaining a compact device design, and can leverage the existing classical communications technology base. Additionally, time-frequency encodings benefit from a relative insensitivity to inhomogeneities in transmission mediums [12,14]. These advantages have been recognized in works exploring the preparation of time-frequency entangled states [15-18], including their use in the violation of Bell inequalities [19,20], quantum key distribution [21], teleportation [22], and continuousvariable cluster states [23].

Quantum computing based on time-frequency encoding has received comparatively little attention, but has become increasingly feasible with the advent of fast switchable integrated phase controllers $[24,25]$. This was highlighted by a recent classical simulation of a quantum random walk based on a time-bin encoding and fast polarization switching [26]. Previous studies have explored unitary operations for time [27,28] and frequency encodings [29], but these implementations have relied on conversion from timefrequency to multiple spatial modes for manipulation.

Here, we present a concept for linear optical quantum computing using time-bin-encoded qubits and only a single spatial mode. Time bins provide a practical solution for the manipulation and detection of time-frequency modes with current technology. We outline methods that provide a sufficient set of operations to allow for universal quantum computing with the KLM scheme. In order to illustrate our scheme, we demonstrate, experimentally, the first implementation of a two-qubit quantum gate in a single spatial mode and show its high fidelity of operation.

Scheme.-We consider a string of time-bin-encoded qubits in a single spatial mode. The polarization degree of freedom is used to define a "register" polarization, in which qubits are stored and transmitted, and a "processing" polarization in which specific time bins are briefly manipulated. After each processing stage, all qubits are returned to the register polarization to ensure that a high degree of coherence is maintained between the time bins during further transmission.

Five basic operations are needed for our implementation, as shown in Fig. 1: a polarization rotation moves a time bin between register and processing polarizations; a displacement operation moves a time bin in the processing polarization forward and backward relative to time bins in the register polarization; a phase shift adds a specified phase between two polarizations; a polarization coupling operation is a partial polarization rotation between two orthogonally polarized time bins; and finally, a readout operation measures the number of photons in a specified bin. With the exception of readout, each of these operations are equivalent to a relative phase shift between appropriate 


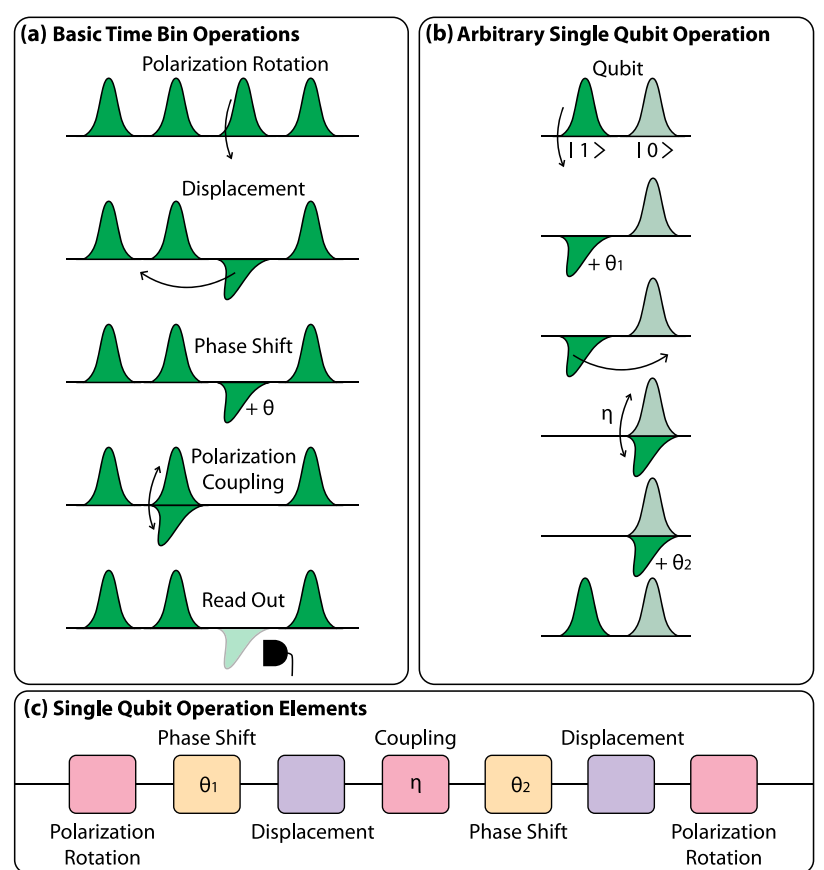

FIG. 1 (color online). (a) Complete set of basic operations necessary for the manipulation of a string of time bins in a single spatial mode. States are initially temporally encoded in the register polarization, shown as vertical. The first operation rotates a time bin to the horizontal processing polarization in order to enable subsequent manipulations as required. After manipulation, the time bins are rotated back into the register polarization in order to protect against dephasing. (b) Operations sufficient for arbitrary single-qubit operations. For brevity, the final displacement and rotation are implicit in the last line. (c) The minimal set of elements required to implement these single-qubit operations.

choices of polarization axes. However, it is convenient to consider them separately here for clarity.

Using this set of manipulations, we show in Fig. 1 how to perform arbitrary single-qubit operations. The operation uses a polarization coupling, equivalent to a variable beamsplitter between the two polarizations, and two relative phase shifts applied to one polarization. It is well known that this is sufficient for local operations on a single qubit [30].

In Fig. 2, we provide a sequence of operations to perform a time-bin-heralded KLM controlled-PHASE (CPHASE) gate $[4,31]$ using two ancilla photons, sufficient to realize the entire KLM scheme in combination with single-qubit operations [1]. This can be trivially combined with local operations to perform a heralded controlled-NOT gate. The proposed scheme could be implemented using four of the sets of the elements in Fig. 1. Alternatively, since each stage of the operation returns the qubits to a single mode and polarization, the string could simply be sent through the same processing elements four times. In this way, the simple set of elements shown could be used to enact arbitrary multigate operations. We observe that our scheme is equally relevant to cluster state computing [2], as it also allows the implementation of type-I and type-II fusion operations [32], suggesting that its utility may extend beyond circuit based quantum computing protocols.

Our scheme is well suited to exploit the readily accessible high dimensionality and robustness of time-frequency modes to environmental dephasing noise. Fast switchable elements can enact different transformations on multiple time bins in a single pass, potentially allowing a substantial reduction in the required number of physical circuit elements. These advantages suggest that this scheme would naturally complement near-deterministic single photon sources $[13,33]$, for which significant challenges exist in building many identical sources. In this case, a single repetitive source would prepare the computational resource state: a string of otherwise indistinguishable single photons in pure quantum states consisting of multiple time bins of a single spatial mode. Our scheme then circumvents the complexity and spatial requirements involved in converting many temporally encoded photons into a spatial encoding. Further, combining temporal and spatial degrees of freedom may enable a significant increase in information capacity [34].

Implementation.-Here, we elaborate on a specific practical implementation of our scheme and discuss its feasibility within the current state of the art. As mentioned above, the basic logical operations are equivalent to a

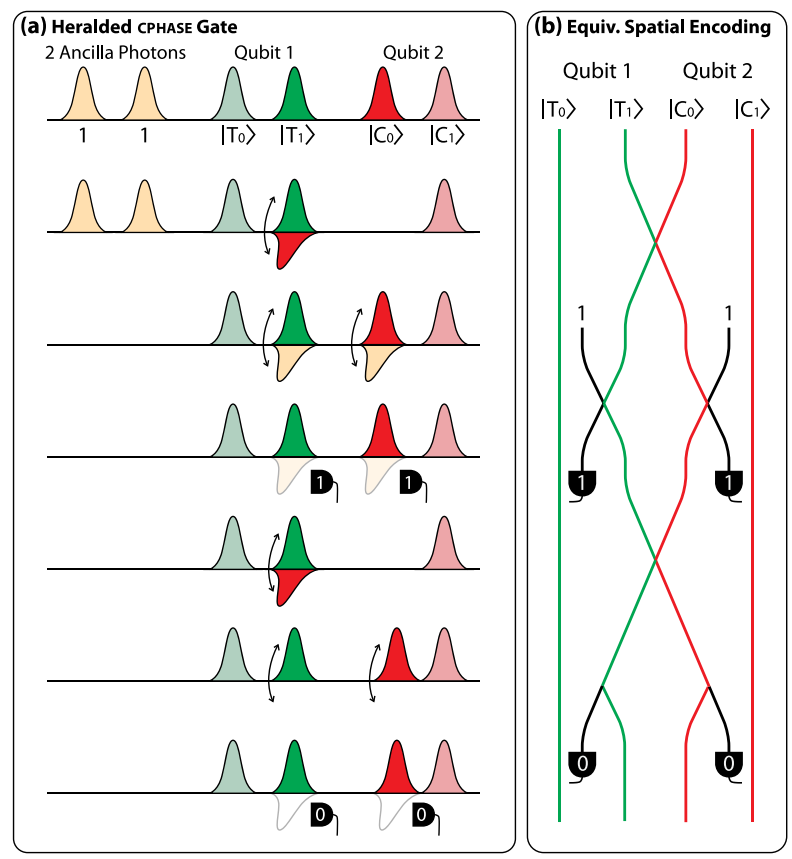

FIG. 2 (color online). (a) Scheme for heralded KLM CPHASE gate using two ancilla photons. Note that these ancilla photons are not encoded as qubits, and each occupies a single time bin. Displacement and rotation operations are omitted for brevity. The numbers on detectors represent the number of photons detected in order to herald successful gate operation. This set of operations could be enacted by four sets of the elements shown in Fig. 1(c), along with appropriate readout elements. (b) Equivalent spatial scheme. Lone numbers represent input ancilla photons. 
relative optical path length difference between a suitable choice of polarization axes. The appropriate experimental approach to generating these path length differences will depend on the specific time-bin structure that is used, as the bandwidth of different photon sources, and, thus, the timebin duration, can differ by several orders of magnitude [35]. Here, we will consider time bins with sub-ps duration; these are suitable for heralded single photons from spontaneous parametric down-conversion pumped by a pulsed laser. Bin-to-bin delay is set by the pump-pulse repetition period, which has been reduced below $10 \mathrm{ps}$ in a number of systems [36-38].

Polarization rotation and polarization coupling operations require a programmable birefringent element that independently manipulates each time bin. The switching time for this element must be less than the delay between consecutive time bins. A suitable integrated optical switch based on cross-phase modulation in a fiber has demonstrated a switching window of 10 ps [24]. As cross-phase modulation is polarization sensitive, this technique could be adapted to create fast-switched birefringent elements.

The detector time resolution does not constrain the binto-bin delay since switching allows arbitrary time-bin components to be moved to the processing polarization or even to a separate readout spatial mode for detection. Therefore, readout can be achieved with standard photonnumber resolving detectors, including spatially $[39,40]$ and temporally multiplexed [41,42] single-photon detectors as well as transition edge sensors $[43,44]$.

For a displacement operation, a simple approach is to use a birefringent element that effects a polarizationdependent path length difference equal to integer multiples of the time-bin separation. A few-cm length of calcite would achieve a displacement of 10 ps. Alternatively, a delay loop could be used, coupled to the main mode by a Mach-Zehnder interferometer. A $\pi$ phase shift created in this interferometer for only one polarization would couple that polarization into the delay line. The controllable phase shift could then be set to keep this polarization in the delay loop for an arbitrary integer number of loops, delaying it with respect to the primary set of time bins. A $3 \mathrm{~mm}$ delay line, possibly implemented on an integrated photonic chip, would create a $10 \mathrm{ps}$ displacement. Although the scheme is no longer entirely single spatial mode with the use of a delay line, the arbitrary number of delay steps it allows may be desirable for faster processing. Finally, in the near future, it should be possible to use a quantum memory to reorder time bins arbitrarily, as demonstrated with classical pulses in a warm-vapor gradient echo memory [45]. This could provide a significant reduction in the number of individual operations needed.

Experiment.-In order to demonstrate the feasibility of our scheme, we have built an entirely single-spatial-mode postselected CPHASE gate for time-encoded qubits [46]. Our gate is equivalent in principle to previous implementations $[6,47]$ that use spatial encoding, often along with a second degree of freedom such as polarization. Preceding the gate is a polarization-to-time conversion stage, and following it a time-to-polarization conversion stage allows for measurement. The experimental layout is shown in Fig. 3. At the core of our experiment, a single-spatial-mode gate is enacted. In this proof-of-principle experiment, we have replaced birefringent switches with passive beam splitters and a second spatial mode, as this allows us to readily incorporate two-mode analogs of single-spatial-mode single-qubit rotations and displacement operations (Fig. 1).

Two spontaneous parametric down-conversion (SPDC) pair sources are used to provide two heralded pure single photons [48]. Initially, one qubit is encoded in the
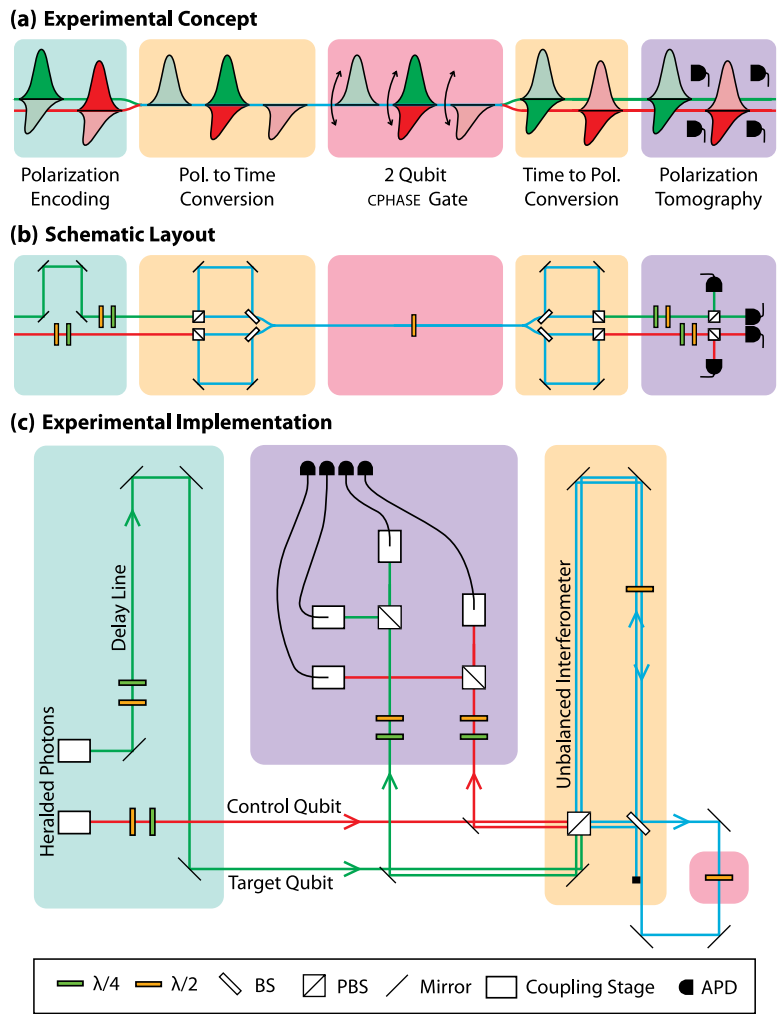

FIG. 3 (color online). (a) Concept for a single-spatial-mode CPHASE gate with preceding state-preparation and following measurement stages. The photons are spectrally degenerate, and are color coded here for clarity. (b) Schematic of the associated experimental layout. Wave plates are used to encode polarization states for both the target photon (green) and the control photon (red). The target photon is delayed with respect to the control photon, and both are coupled into unbalanced interferometers for conversion of polarization encoding to time encoding. The photons are then combined into a single spatial mode in which a twoqubit gate is implemented using a half-wave plate. Conversion back to polarization-encoding states again uses unbalanced interferometers. Finally, polarization tomography is carried out using four avalanche-photodiode (APD) detectors. (c) Actual experimental implementation. Two SPDC sources provide heralded single photons for the experiment, which proceeds as described above, except that a single unbalanced interferometer is used instead of the four separate unbalanced interferometers for conversion between polarization-encoding and time-encoding. BSbeam splitter; PBS-polarizing beam splitter. 
polarization of each photon. The qubits are then converted to a time basis using an unbalanced interferometer, producing two orthogonally polarized photons in a common spatial mode. One of the photons is delayed so that its first time bin coincides with the second time bin of the other photon. The gate operation is implemented by using a half wave plate to couple the polarizations, resulting in Hong-Ou-Mandel interference [49] between the two coincident time bins and allowing a postselected nonlinear interaction.

To characterize the two-photon operation of our gate, we initially input a control photon with a horizontal $(H)$ or vertical $(V)$ polarization and a target photon with an antidiagonal $(A)$ or diagonal $(D)$ polarization. For these inputs, the CPHASE gate should swap the target photon polarization between $A$ and $D$ if the control photon is $V$ polarized. The measured gate outcomes are shown in Fig. 4, where the control and target photons are measured in the $H-V$ and $A-D$ bases, respectively. For these bases, we define a classical fidelity measure [50]

$$
\begin{aligned}
F_{H A}= & \frac{1}{4}[P(H A \mid H A)+P(H D \mid H D) \\
& +P(V D \mid V A)+P(V A \mid V D)],
\end{aligned}
$$

where, for example, $P(V A \mid V D)$ represents the conditional probability of measuring outputs $V$ and $A$ given input $V$ and $D$ for the control and target photons, respectively. We measure a classical fidelity of $F_{H A}=0.84 \pm 0.03$. Changing the photon inputs to the control $A-D$ and target $H-V$ bases and also measuring in these bases, equivalent to transforming the bases by a Hadamard operation, allows us to measure a complementary fidelity $F_{A H}$. For this latter case, we measure a similar fidelity $F_{A H}=0.84 \pm 0.02$.

Following [50], we use these fidelity measures to bound the quantum process fidelity. The resulting bound of the gate process fidelity $F_{\text {process }}$

$$
F_{A H}+F_{H A}-1 \leq F_{\text {process }} \leq \operatorname{Min}\left[F_{A H}, F_{H A}\right]
$$

is calculated to be $0.68 \pm 0.04 \leq F_{\text {process }} \leq 0.84 \pm 0.02$, comparable to other optical two-qubit gate implementations $[6,51]$.

An alternative measure of our gate fidelity demonstrates its nonclassical operation. For this, we consider an additional choice of bases with both inputs in the $A-D$ basis, and both outputs in the $R-L$ (right-left) basis. We measure the classical fidelity for this operation to be $F_{A A}=0.85 \pm$ 0.06 . As shown in [52], since this measure, along with $F_{H A}$ and $F_{A H}$, are all greater than $2 / 3$, the gate operation must be nonclassical. Our gate exceeds this criterion with $99.8 \%$ confidence.

The gate fidelity is limited by the spatial mode overlap of our photons. Because of the long path length in the time-to-polarization converter, this overlap is sensitive to the slight changes in alignment caused by temperature variations and vibrations. This path length is necessary to achieve a delay between consecutive time bins that is resolvable by the coincidence counting (a)

Experiment

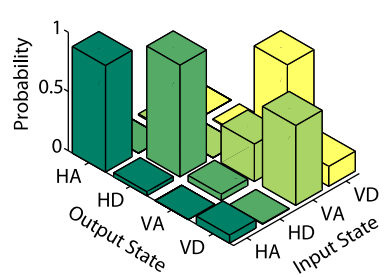

(b)

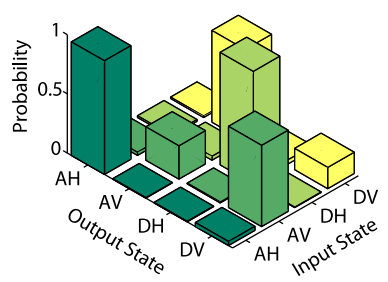

(c)

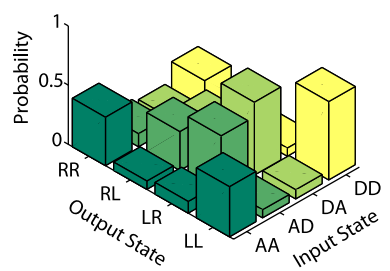

Theory
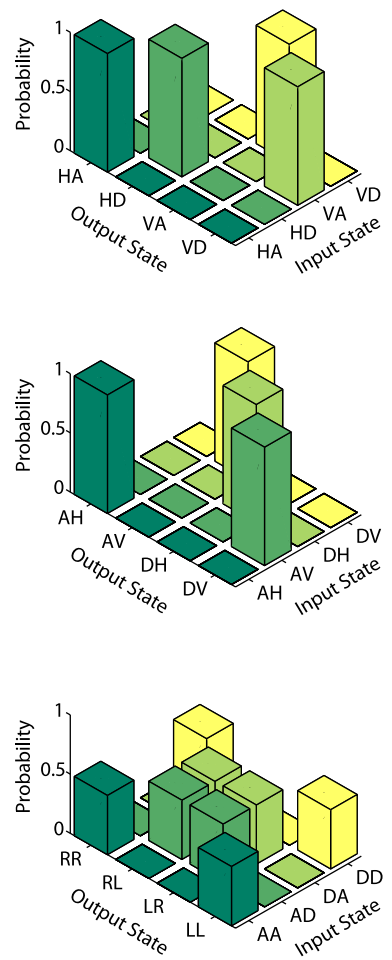

FIG. 4 (color online). Two-qubit output state measurements: (a) Input $H-V$ and $A-D$ bases for control and target photons, respectively, output $H-V$ and $A-D$ bases. The measured classical fidelity for this operation is $F_{H A}=0.84 \pm 0.03$. (b) Input $A-D$ and $H-V$ bases, output $A-D$ and $H-V$ bases, resulting in $F_{A H}=$ $0.84 \pm 0.02$. (c) Input $A-D$ bases for both photons, output $R-L$ bases for both photons, resulting in $F_{A A}=0.85 \pm 0.06$. Theoretical ideal outputs are shown for comparison.

electronics and detectors [46]. We modeled this effect by calculating the ideal gate operation on partially distinguishable input photons in the states $|\psi\rangle$ and $\alpha|\psi\rangle+$ $\sqrt{1-\alpha^{2}}\left|\psi_{\text {disting. }}\right\rangle$, respectively, and found that $\alpha=0.91$ minimized the L1 distance between the results and theoretical predictions.

Conclusions.-We have presented a scheme for linear optical quantum computing using time-bin-encoded qubits in a single spatial mode. We have shown how to implement arbitrary single-qubit operations and a heralded CPHASE gate as required for universal quantum computing in the KLM scheme. In support of this concept, we have demonstrated a novel post-selected single-spatial-mode two-qubit CPHASE gate. We measured an average classical gate fidelity of $0.84 \pm 0.07$ across three different bases, confirming its nonclassical operation.

We thank J. Nunn for helpful discussions. This work was supported by the Engineering and Physical Sciences Research Council (Contract No. EP/H03031X/1), the European Commission Project Q-ESSENCE (No. 248095) and the Air Force Office of Scientific

Research (European Office of Aerospace Research and Development). 
[1] E. Knill, R. Laflamme, and G. J. Milburn, Nature (London) 409, 46 (2001).

[2] P. Kok, W. J. Munro, K. Nemoto, T. C. Ralph, J.P. Dowling, and G. J. Milburn, Rev. Mod. Phys. 79, 135 (2007).

[3] T. C. Ralph and G. J. Pryde, in Progress in Optics, edited by E. Wolf (Elsevier, New York, 2010), Vol. 54, pp. 209-269.

[4] T. Pittman, B. Jacobs, and J. Franson, Phys. Rev. A 64, 062311 (2001).

[5] S. Gasparoni, J.-W. Pan, P. Walther, T. Rudolph, and A. Zeilinger, Phys. Rev. Lett. 93, 020504 (2004).

[6] J. L. O'Brien, G. J. Pryde, A. G. White, T. C. Ralph, and D. Branning, Nature (London) 426, 264 (2003).

[7] X.-H. Bao, T.-Y. Chen, Q. Zhang, J. Yang, H. Zhang, T. Yang, and J.-W. Pan, Phys. Rev. Lett. 98, 170502 (2007).

[8] B. P. Lanyon, M. Barbieri, M. P. Almeida, T. Jennewein, T. C. Ralph, K. J. Resch, G. J. Pryde, J.L. O’Brien, A. Gilchrist, and A. G. White, Nat. Phys. 5, 134 (2008).

[9] R. Okamoto, J.L. O'Brien, H.F. Hofmann, and S. Takeuchi, Proc. Natl. Acad. Sci. U.S.A. 108, 10067 (2011).

[10] X.-S. Ma, S. Zotter, J. Kofler, T. Jennewein, and A. Zeilinger, Phys. Rev. A 83, 043814 (2011).

[11] H. de Riedmatten, I. Marcikic, V. Scarani, W. Tittel, H. Zbinden, and N. Gisin, Phys. Rev. A 69, 050304 (2004).

[12] A. Hayat, X. Xing, A. Feizpour, and A. M. Steinberg, Opt. Express 20, 29174 (2012).

[13] P. B. R. Nisbet-Jones, J. Dilley, A. Holleczek, O. Barter, and A. Kuhn, New J. Phys. 15, 053007 (2013).

[14] R. Thew, S. Tanzilli, W. Tittel, H. Zbinden, and N. Gisin, Phys. Rev. A 66, 062304 (2002).

[15] J. Brendel, N. Gisin, W. Tittel, and H. Zbinden, Phys. Rev. Lett. 82, 2594 (1999).

[16] C. Simon and J.-P. Poizat, Phys. Rev. Lett. 94, 030502 (2005).

[17] J. Barreiro, N. Langford, N. Peters, and P. Kwiat, Phys. Rev. Lett. 95, 260501 (2005).

[18] A. Zavatta, M. D’Angelo, V. Parigi, and M. Bellini, Phys. Rev. Lett. 96, 020502 (2006).

[19] J. Franson, Phys. Rev. Lett. 62, 2205 (1989).

[20] L. Olislager, J. Cussey, A. T. Nguyen, P. Emplit, S. Massar, J.-M. Merolla, and K.P. Huy, Phys. Rev. A 82, 013804 (2010).

[21] W. Tittel, J. Brendel, H. Zbinden, and N. Gisin, Phys. Rev. Lett. 84, 4737 (2000).

[22] I. Marcikic, H. de Riedmatten, W. Tittel, H. Zbinden, and N. Gisin, Nature (London) 421, 509 (2003).

[23] N. C. Menicucci, Phys. Rev. A 83, 062314 (2011).

[24] M. A. Hall, J. B. Altepeter, and P. Kumar, Phys. Rev. Lett. 106, 053901 (2011).

[25] D. Bonneau, M. Lobino, P. Jiang, C. Natarajan, M. Tanner, R. Hadfield, S. Dorenbos, V. Zwiller, M. Thompson, and J. O’Brien, Phys. Rev. Lett. 108, 1 (2012).

[26] A. Schreiber, A. Gábris, P. P. Rohde, K. Laiho, M. Štefaňák, V. Potoček, C. Hamilton, I. Jex, and C. Silberhorn, Science 336, 55 (2012).

[27] Y. Soudagar, F. Bussières, G. Berlín, S. Lacroix, J. M. Fernandez, and N. Godbout, J. Opt. Soc. Am. B 24, 226 (2007).
[28] F. Bussieres, Y. Soudagar, G. Berlin, S. Lacroix, and N. Godbout, arXiv:quant-ph/0608183.

[29] E. Huntington and T. Ralph, Phys. Rev. A 69, 042318 (2004).

[30] R. Simon and N. Mukunda, Phys. Lett. A 143, 165 (1990).

[31] T. Ralph, A. White, W. Munro, and G. Milburn, Phys. Rev. A 65, 012314 (2001).

[32] See Supplemental Material at http://link.aps.org/ supplemental/10.1103/PhysRevLett.111.150501 for a description of one possible method for the implementation of fusion gates in our scheme.

[33] N. Lindner and T. Rudolph, Phys. Rev. Lett. 103, 113602 (2009).

[34] J. T. Barreiro, T.-C. Wei, and P. G. Kwiat, Nat. Phys. 4, 282 (2008).

[35] M. D. Eisaman, J. Fan, A. Migdall, and S. V. Polyakov, Rev. Sci. Instrum. 82, 071101 (2011).

[36] A.E.H. Oehler, M.C. Stumpf, S. Pekarek, T. Südmeyer, K. J. Weingarten, and U. Keller, Appl. Phys. B 99, 53 (2010).

[37] B. Dromey, M. Zepf, M. Landreman, K. O'keeffe, T. Robinson, and S. M. Hooker, Appl. Opt. 46, 5142 (2007).

[38] K. G. Wilcox, A. H. Quarterman, V. Apostolopoulos, H. E. Beere, I. Farrer, D. A. Ritchie, and A. C. Tropper, Opt. Express 20, 7040 (2012).

[39] S. Jahanmirinejad, G. Frucci, F. Mattioli, D. Sahin, A. Gaggero, R. Leoni, and A. Fiore, Appl. Phys. Lett. 101, 072602 (2012).

[40] A. Divochiy, F. Marsili, D. Bitauld, A. Gaggero, R. Leoni, F. Mattioli, A. Korneev, V. Seleznev, N. Kaurova, O. Minaeva, G. Gol'tsman, K. G. Lagoudakis, M. Benkhaoul, F. Lévy, and A. Fiore, Nat. Photonics 2, 302 (2008).

[41] M. Fitch, B. Jacobs, T. Pittman, and J. Franson, Phys. Rev. A 68, 043814 (2003).

[42] D. Achilles, C. Silberhorn, C. Sliwa, K. Banaszek, and I. A. Walmsley, Opt. Lett. 28, 2387 (2003).

[43] T. Gerrits, N. Thomas-Peter, J. Gates, A. Lita, B. Metcalf, B. Calkins, N. Tomlin, A. Fox, A. Linares, J. Spring, N. Langford, R. Mirin, P. G. Smith, I. Walmsley, and S. Nam, Phys. Rev. A 84, 1 (2011).

[44] A. Lamas-Linares, B. Calkins, N. A. Tomlin, T. Gerrits, A. E. Lita, J. Beyer, R. P. Mirin, and S. W. Nam, Appl. Phys. Lett. 102, 231117 (2013).

[45] M. Hosseini, B. M. Sparkes, G. Hétet, J. J. Longdell, P. K. Lam, and B. C. Buchler, Nature (London) 461, 241 (2009).

[46] See Supplemental Material at http://link.aps.org/ supplemental/10.1103/PhysRevLett.111.150501 for further details on the experimental method.

[47] T. Ralph, N. Langford, T. Bell, and A. White, Phys. Rev. A 65, 062324 (2002).

[48] P. Mosley, J. Lundeen, B. Smith, P. Wasylczyk, A. U'Ren, C. Silberhorn, and I. Walmsley, Phys. Rev. Lett. 100, 133601 (2008).

[49] C. K. Hong, Z. Y. Ou, and L. Mandel, Phys. Rev. Lett. 59, 2044 (1987).

[50] H. F. Hofmann, Phys. Rev. Lett. 94, 160504 (2005).

[51] N. Langford, T. Weinhold, R. Prevedel, K. Resch, A. Gilchrist, J. O'Brien, G. Pryde, and A. White, Phys. Rev. Lett. 95, 210504 (2005).

[52] H. Hofmann, Phys. Rev. A 72, 022329 (2005). 